\title{
ATENDIMENTO PSICOLÓGICO NUMA UNIDADE DE TRANSPLANTE DE MEDULA ÓSSEA
}

\author{
PSYCHOLOGICAL SERVICE IN A BONE MARROW TRANSPLANTATION UNIT
}

Luciana Marchetti Torrano-Masetti ${ }^{1}$; Érika Arantes de Oliveira ${ }^{2}$ \& Manoel Antônio dos Santos ${ }^{3}$

\begin{abstract}
${ }^{1}$ Psicóloga da Unidade de Transplante de Medula Óssea e da Clínica de Hematologia do Hospital das Clínicas da Faculdade de Medicina de Ribeirão Preto - USP, Mestranda; ${ }^{2}$ Psicóloga voluntária da Unidade de Transplante de Medula Óssea do Hospital das Clínicas da Faculdade de Medicina de Ribeirão Preto - USP, Mestranda; ${ }^{3}$ Docente do Departamento de Psicologia e Educação da Faculdade de Filosofia, Ciências e Letras de Ribeirão Preto - USP, coordenador do NEPP - Núcleo de Ensino e Pesquisa em Psicologia Clínica. CoRReSPondênCIA: Luciana Marchetti Torrano-Masetti - Rua Cerqueira César, 880/32, Centro - 14010-130 - Ribeirão Preto - SP. E-mail: agrot@neomundi.com.br
\end{abstract}

TORRANO-MASETTI LM; OLIVEIRA EA \& SANTOS MA. Atendimento psicológico numa unidade de transplante de medula óssea. Medicina, Ribeirão Preto, 33: 161-169, abr./Jun. 2000.

RESUMO: O Transplante de Medula Óssea (TMO) vem se constituindo como uma alternativa terapêutica eficaz, em diversos tipos de neoplasias e doenças hematológicas, quando os tratamentos convencionais não oferecem bom prognóstico. Trata-se de um procedimento complexo tanto do ponto de vista físico como psicológico, de modo que a necessidade de uma intervenção psicológica junto aos pacientes e familiares tem sido amplamente reconhecida na atualidade. Segundo a literatura da área, a importância do acompanhamento psicológico não se restringe somente à internação, devendo abarcar também o pré e o pós-TMO, incluindo, no processo de intervenção, os familiares. Levando-se em consideração tais constatações, foi estruturado o serviço de atendimento psicológico da UTMO do HCFMRP-USP. O objetivo do presente trabalho é descrever a estruturação do serviço, bem como apresentar os principais achados de estudos de avaliação realizados para se determinar a efetividade de algumas modalidades de intervenção psicológica implementadas. Os resultados obtidos corroboram dados disponíveis na literatura, que apontam a necessidade de uma intervenção, junto aos pacientes, anterior à internação, um apoio psicológico durante as diferentes fases da internação e o acompanhamento psicológico ao familiar.

UNITERMOS: Psicologia Aplicada. Apoio Social. Transplante de Medula Óssea.

\section{1- INTRODUÇÃO}

O Transplante de Medula Óssea (TMO) vem se constituindo, atualmente, como uma alternativa eficaz em diversos tipos de neoplasias e doenças hematológicas, quando os tratamentos convencionais não oferecem bom prognóstico.

Essa modalidade terapêutica tem sido realizada na Unidade de Transplante de Medula Óssea, do Hospital das Clínicas da Faculdade de Medicina de Ribeirão Preto, desde 1993, através do trabalho de uma equipe multidisciplinar, composta pelos membros da enfermagem, pela equipe médica, por uma assistente social, uma nutricionista, uma fisioterapeuta e duas psicólogas.

A inclusão do psicólogo nas equipes de cuidados oncológicos está relacionada diretamente com o reconhecimento da interação existente entre aspectos físicos e psicológicos, o que acabou por conduzir à estruturação de abordagens multidisciplinares para a administração das manifestações sintomatológicas que esses pacientes podem desenvolver. 
Trata-se de um fenômeno muito recente em nosso meio ${ }^{(1)}$. A partir do início da década de 90 é que houve uma grande expansão na inserção dos profissionais de psicologia, sendo que, na atualidade, muitos hospitais, espalhados por todo o Brasil, contam com a colaboração do serviço psicológico ${ }^{(2)}$.

Tal expansão pode ser vislumbrada através da publicação de diversos relatos de trabalhos realizados nessa área, em vários estados brasileiros ${ }^{(2)}$. Dentre os relatos, pode-se destacar a descrição do serviço de psiconcologia do Hospital Israelita Albert Einstein ${ }^{(3)}$, de São Paulo. Implantado em 1991, o serviço também é desenvolvido em uma Unidade de Transplante de Medula Óssea. O serviço engloba um conjunto de estratégias para a abordagem do paciente e de seus familiares, tais como: esclarecimento e orientação a pacientes e/ou familiares; intervenções psicossociais segundo o modelo de psicoterapia breve em crise; atendimentos familiares; discussão de casos clínicos; grupos de vivência; grupo aberto para os acompanhantes; grupos fechados para pacientes oncológicos em radioterapia e quimioterapia; grupo aberto na sala de espera; atividades ocupacionais, produção de material educativo e terapêutico; orientação para a aquisição de complementos específicos (perucas, próteses, etc.); projetos de pesquisa e estudos científicos; atendimento psicossocial à equipe.

Constata-se, através dessa descrição, que é amplo o papel do profissional de psicologia nesse contexto. A sua intervenção inicia-se antes da internação e não se restringe somente ao paciente, estendendo-se ainda aos familiares, acompanhantes e profissionais da equipe.

A estruturação de serviço é muito semelhante à da Unidade de Transplante de Medula Óssea (UTMO), do Hospital das Clínicas da Faculdade de Medicina de Ribeirão Preto (SP), que visa intervir junto ao paciente, seus familiares, sem esquecer o cuidador ("acompanhante") e, mais recentemente, o doador da medula óssea. Essa intervenção inicia-se na etapa que precede o transplante (pré-TMO), inclui o período de internação para a efetuação do procedimento (TMO) e, finalmente, preocupa-se em contribuir com a reabilitação psicossocial do paciente após o TMO.

\section{2- OBJETIVO}

O presente trabalho tem como objetivo descrever a estruturação do serviço nos últimos dois anos (1998-99), bem como apresentar os resultados de es- tudos que objetivaram avaliar a efetividade de algumas modalidades de intervenção implementadas. Através dessa descrição, é possível compreender a intervenção psicológica como uma atuação clínica indissociável da pesquisa, inserida dentro de um contexto multidisciplinar de atendimento.

\section{3- ATENDIMENTO PSICOLÓGICO NA UNI- DADE DE TMO}

\subsection{Fase que precede o TMO}

A importância de uma intervenção, antes da realização do transplante de medula óssea, advém da constatação de que dois momentos que antecedem o procedimento propriamente dito podem desencadear sofrimento e conflitos psicológicos: são os momentos da tomada de consciência do diagnóstico e da opção pela realização do TMO. Por outro lado, esses momentos são fundamentais para a tomada de decisão frente ao tratamento e às formas de enfrentamento da enfermidade.

O impacto psicológico do momento do diagnóstico atinge, além do paciente, toda a unidade familiar ${ }^{(4)}$, uma vez que o paciente provém de um núcleo social primário, que sofrerá as implicações da sua situação de doença ${ }^{(5)}$.

A descoberta de uma doença com um prognóstico tão reservado, como é o câncer, traz consigo o temor frente à possibilidade da morte, sendo que, para muitos, essa possibilidade transforma-se em certeza e, conseqüentemente, o diagnóstico converte-se em sentença de morte.

A família merece, nesse instante, um cuidado especial, uma vez que o momento tem um enorme impacto sobre os familiares, que vêem seu mundo desabar após o diagnóstico ${ }^{(5)}$. Isso faz com que, em muitas circunstâncias, as suas necessidades psicológicas excedam as do paciente, dependendo da intensidade das reações emocionais desencadeadas, tornando a ansiedade familiar um dos aspectos de muito difícil administração $\mathrm{O}^{(6)}$.

Se, por algum motivo, essa etapa do diagnóstico não transcorrer da forma esperada (uma não aceitação da doença, por exemplo), poderão advir problemas para o paciente ${ }^{(7)}$ : complicações médicas (exacerbação dos sintomas físicos, aceleração no avançar do câncer); sociais (problemas maritais, diminuição do status socioeconômico); psicológicas e psiquiátricas (maior ansiedade, abuso de álcool, enfrentamento pouco efetivo da doença). Fica evidente aqui 
a necessidade da intervenção de um profissional de saúde mental para oferecer suporte emocional a esses pacientes.

Passado o momento do diagnóstico, tem-se a opção do TMO e um novo conflito se instala: realizar ou não tal procedimento? Esse questionamento aparece principalmente em virtude das peculiaridades dessa terapêutica, vista como um tratamento salvador e, ao mesmo tempo, um tratamento ameaçador ${ }^{(8)}$.

Nessa última categoria (o tratamento como uma ameaça), o transplante carrega consigo riscos pessoais severos para o paciente (ameaça da perda da integridade física e, acima de tudo, da própria vida). Paralelamente, essas ameaças afetam também a família do paciente devido à possibilidade palpável de se perder um ente querido ${ }^{(9)}$.

Seguindo os dados da literatura, associados às demandas específicas da Unidade de TMO, foi desenvolvido um plano de intervenção para a fase préTMO, que engloba os procedimentos relacionados a seguir.

\subsubsection{Avaliação psicológica com o paciente}

Quando o paciente aceita submeter-se ao tratamento, ele passa por um processo de avaliação psicológica, que se destina principalmente a:

1) verificar a existência de alguma contra-indicação ao transplante na presença de uma doença psiquiátrica grave;

2) conhecer o sujeito, sua estrutura psicossocial, como ele vive e gosta de viver, o que lhe dá prazer, suas expectativas frente ao tratamento, suas ansiedades, medos e dúvidas;

3) formular o prognóstico quanto ao nível adaptativo (como poderá se adaptar e suportar as restrições do pós-transplante);

4) perceber como o paciente reage às informações médicas, que aspectos ele prioriza e por quê;

5) compreender a situação familiar e sua rede social de apoio, bem como os papéis que o paciente desempenha como membro da família;

6) verificar a qualidade do relacionamento interpessoal entre paciente e doador;

7) discriminar que tipo de orientações serão necessárias e qual a melhor maneira de transmiti-las para a família e o acompanhante no período de internação e nos cem dias de hospital-dia.

A obtenção dessas informações pormenorizadas é essencial para o planejamento da intervenção psicológica no enfrentamento, adaptação e aderência ao tratamento médico.

\subsubsection{Avaliação com o doador}

Há necessidade de verificação das percepções dos doadores a respeito do procedimento, uma vez que grande parte deles têm concepções errôneas sobre a doação ${ }^{(10)}$. É fundamental que tudo se esclareça antes de iniciado o condicionamento do receptor, de modo a garantir a doação, uma vez que o paciente, após receber uma dose letal de drogas, necessitará obrigatoriamente da infusão da medula do doador.

Além dessa preocupação com a saúde do receptor, a avaliação do doador visa auxiliá-lo, quando necessário, uma vez que estudos comprovam que a questão da doação traz consigo muitas implicações psicológicas para os doadores. Essas manifestam-se antes da doação, muitas vezes sob a forma de angústia; imediatamente depois, através da influência dos fatores psicológicos na percepção do procedimento de doação; decorridos alguns anos, através da constatação de modificações em sua qualidade de vida.

\subsubsection{Grupos de pacientes em fila de espera}

Após o paciente receber a indicação para o TMO, ele deve comparecer às consultas médicas, executar os exames e ter um doador. Assim que ele recebe as informações médicas sobre o processo, ele toma conhecimento de que não só o enxerto da medula é importante, mas todos os fatores implicados no processo (condicionamento, pega da medula, doença do enxerto contra o hospedeiro - DECH, mucosite, infecções, riscos, estado nutricional, físico, psicológico, familiar, de higiene, autocuidados, etc.). O paciente é exposto aos mais variados tipos de sentimentos e reações que, aliados a seus desejos, mediarão suas decisões e o encaminhamento do seu tratamento, o que se reflete diretamente em sua vida.

A inserção de um grupo pré-TMO visa promover a saúde mental através da ampliação da possibilidade de troca entre os pacientes e da inclusão de um espaço preservado para a verbalização e compartilhamento de suas vivências. Os grupos são coordenados por uma psicóloga especializada, que diferentemente dos amigos e familiares, está capacitada a ouvilos em seu sofrimento. Essa escuta qualificada se dá em uma outra perspectiva, que a separa de outras modalidades de contato social. Assim, cria-se uma atmosfera emocional propícia para se tratar de questões cruciais como dúvidas em relação à efetividade do tratamento, as angústias mobilizadas pela espera sem uma garantia prévia de um desfecho bem-sucedido. Subjacente a essas questões, a própria morte é colocada em perspectiva. 
Nesses encontros, os pacientes têm a possibilidade de incluírem, em seu repertório, questões levantadas por seus companheiros, com as quais não haviam se preocupado até então, ampliando assim seu conhecimento e suas possibilidades de administração das situações difíceis.

Com o objetivo de avaliar os efeitos e possíveis benefícios dessa modalidade de intervenção, realizou-se um estudo sobre a influência do grupo préTMO sobre a adaptação inicial e o ajustamento psicossocial pós-TMO ${ }^{(11)}$. Foram entrevistados 10 pacientes, de ambos os sexos, com idades variando entre 22 e 46 anos, que se submeteram ao transplante alogênico no HC-FMRP-USP há, no máximo, 12 meses e se encontravam em seguimento ambulatorial.

Os pacientes foram distribuídos randomicamente em dois grupos, sendo um composto por quatro pacientes que participaram do grupo preparatório (grupo experimental: GE), e o outro composto por seis pacientes cujo preparo não incluiu esse procedimento (grupo controle: GC). O instrumento utilizado para a coleta de dados foi uma entrevista semidirigida, aplicada individualmente, em situação face a face. As entrevistas foram gravadas em áudio e, posteriormente, transcritas na íntegra. O tratamento dos dados compreendeu uma análise temática do material, realizada independentemente por dois examinadores, com o objetivo de extrair os temas e as unidades de significado que emergiram dos depoimentos.

Os resultados evidenciam que os pacientes do GE mostraram-se mais sensíveis à importância dos aspectos emocionais no enfrentamento do processo de adaptação às diferentes fases do TMO, levantando sentimentos relacionados à "solidão", "tristeza" e necessidade de "tranqüilidade familiar".

Já o grupo controle (GC) tendeu a enfatizar fatores externos que auxiliariam nessa adaptação, tais como: "sorte", "informação" e "apoio da família e/ou do médico". A avaliação do GE denota o alívio resultante da possibilidade de receber previamente informações técnicas sobre o procedimento e poder compartilhar dificuldades com pessoas que atravessam a mesma situação vital, destacando-se os fatores de universalidade, apoio e continência emocional.

Os resultados desse trabalho informam que as orientações e esclarecimentos proporcionados pela experiência do grupo contribuíram para instrumentalizar psicologicamente o paciente, fortalecendo a relação de confiança na equipe e em si próprio.

\subsubsection{Reuniões familiares}

São oferecidas às famílias de pacientes com idade inferior a 21 anos ou quando houver alguma indicação específica.

O objetivo é fornecer suporte emocional e informações sobre os procedimentos hospitalares, promovendo, junto aos familiares, a conscientização sobre os recursos que podem ser mobilizados no enfrentamento da doença, trabalhando as resistências às mudanças e inserindo a unidade familiar como parte integrante do processo terapêutico.

\subsubsection{Atendimento individual do paciente}

É um tipo de acompanhamento que pode ser solicitado por algum profissional da equipe, ou sua necessidade pode ser percebida através dos resultados do procedimento de avaliação psicológica. A preparação do paciente para todo o processo de internação e acompanhamento ambulatorial visa encontrar formas de enfrentamento para que essa experiência não seja vivenciada a priori como terrível ou aniquiladora, e, sim, como favorecedora de possibilidades de vida.

\subsection{Durante o TMO}

A intervenção psicológica durante a internação visa colaborar para a integridade psíquica do paciente, que ainda terá que atravessar outros sete estágios psicológicos, além da decisão de aceitar o $\mathrm{TMO}^{(12)}$ :

\subsubsection{Decisão de realizar o TMO}

O TMO favorece o desencadeamento de reações psicológicas de ambivalência, negação, recusa e ansiedade, suscitadas pelo fato de se tratar de um procedimento de risco, que oferece simultaneamente a possibilidade de prolongar ou abreviar a vida.

\subsubsection{Avaliação da internação e planejamento de cuidados}

A situação da internação exige que o paciente e seus familiares reorganizem sua rotina e seus planos para o futuro, sustentados pela esperança depositada no procedimento. Essa incerteza pode ser administrada através da negação ou do afeto "embotado", uma vez que a vulnerabilidade da condição é muito carregada de ansiedade.

\subsubsection{Regime de isolamento/condicionamento}

A condição do isolamento protetor e a deficiência imunológica, causada pela quimioterapia, eleva 
o nível de ansiedade, precipita o surgimento da dependência e gera preocupações com a morte. A solidão vivenciada no isolamento pode favorecer sentimentos de pesar diante das perdas já sofridas em relação ao papel social e aos relacionamentos afetivos, além de outros prejuízos.

\subsubsection{TMO propriamente dito}

O sentimento aqui manifestado é, geralmente, de gratidão ao doador e de alívio pelo cumprimento de uma etapa do processo. A data do TMO adquire um significado especial. Por exemplo, pode representar o renascimento do paciente, renascimento que é simbolizado pela comemoração de seu "aniversário" nesse dia e não no da data do nascimento; em algumas circunstâncias, os familiares afixam na porta do quarto enfeites semelhantes aos colocados em maternidades para saudar e dar boas-vindas ao recém-nascido.

\subsubsection{Rejeição do enxerto versus pega}

Nessa etapa, existe a possibilidade do não enxertamento da medula, implicando em risco de vida, o que eleva o nível de ansiedade. Aqui são freqüentes os sintomas depressivos, comportamentos regressivos, labilidade emocional, frustração, arrependimentos quanto à decisão de realizar o TMO e, até mesmo, manifestações de desesperança e raiva dirigidas à equipe.

\subsubsection{Desenvolvimento de DECH ou GVHD (graf-versus-hot disease)}

As manifestações emocionais predominantes nessa circunstância são, basicamente, a raiva e a depressão, desencadeadas pela substituição de uma doença por outra.

\subsubsection{Preparação para alta da enfermaria}

A saída da enfermaria é envolta por reações ambivalentes: alívio pela possibilidade de saída do isolamento protetor e insegurança por não se poder contar mais com o amparo e os cuidados da equipe de saúde. A volta ao convívio social, mesmo que restrito, pode despertar sentimentos de desvalia e tristeza, desencadeados pelas alterações e distorções na imagem corporal.

\subsubsection{Adaptação fora do hospital}

A fase de adaptação inicial oferece uma problemática complexa. Pode envolver alterações proprioceptivas e do esquema corporal, déficit cognitivo temporário, disfunções sexuais, problemas psicomo- tores, restrições de hábitos cotidianos (não tomar sol, seguir dieta alimentar, praticar exercícios fisioterápicos, administrar um grande número de medicamentos). Esse conjunto de fatores pode dificultar a reinserção e reabilitação psicossocial.

\subsection{O trabalho do psicólogo na enfermaria do TMO}

\subsubsection{Atendimentos individuais nas enfermarias}

Os atendimentos ocorrem no isolamento, com uma freqüência média de três vezes por semana, sendo que esse número varia em função das especificidades de cada caso.

Foi realizado em um estudo retrospectivo a respeito dos atendimentos psicológicos e psiquiátricos que haviam sido efetuados na UTMO desde o seu início ${ }^{(13)}$. O trabalho buscou verificar a importância da inclusão do profissional de saúde mental em uma equipe de TMO. Para tanto, utilizou-se a análise das anotações realizadas pelos profissionais de saúde mental nos prontuários dos pacientes que haviam se submetido ao TMO, englobando um total de 120 transplantes, sendo 20 autólogos e 100 alogênicos.

Através de leituras minuciosas dos registros contidos nos prontuários médicos, elaborou-se, retrospectivamente, uma caracterização do perfil dos sujeitos e descreveu-se o desenrolar de sua internação e das intervenções dos profissionais de saúde mental: tipo de acompanhamento, motivo da solicitação desses acompanhamentos e procedimentos realizados.

A análise dos dados obtidos indicou que a cada fase do transplante corresponde uma resposta emocional específica dos pacientes, o que justifica a adoção de uma abordagem apropriada a cada tipo de necessidade apresentada, visando preservar sua saúde emocional. Esses resultados aparecem no quadro que se segue.

Através desses resultados pôde-se evidenciar a importância do atendimento psicológico acessível a todos os pacientes desde o início do processo, bem como as necessidade emocionais específicas de cada paciente, que precisam ser corretamente identificadas para que possam ser traçadas estratégias de intervenção mais efetivas.

\subsubsection{Grupo de acompanhantes}

Os pacientes necessitam de um acompanhante (cuidador) durante todo o período de internação, que costuma ser um membro da família. Para colaborar 
com essa tarefa e aprimorar as estratégias de enfrentamento do processo por parte do acompanhante, foi instituído um grupo de apoio, aberto, composto por, no máximo, cinco e, no mínimo, três pessoas, coordenado por uma psicóloga e observado por uma estagiária, com duração de uma hora e freqüência semanal.

Foi realizado um estudo tendo como objetivo descrever o processo grupal e averigüar seus efeitos sobre os acompanhantes ${ }^{(14)}$. Foi estudada uma amostra de 17 acompanhantes $(n=17)$. Procedeu-se a uma análise das transcrições das sessões ocorridas no período de dez meses, constituindo um corpus de 40 sessões. Foi empregada uma metodologia qualitativa de avaliação do processo grupal, aplicando-se uma análise temática aos segmentos dos relatos verbais. $\mathrm{O}$ ma- terial foi submetido à avaliação independente de dois juízes. Essa análise permitiu a elucidação do movimento do grupo e a constatação de alguns "tópicos/temas" que emergiram durante as sessões.

No decorrer dos grupos pôde-se perceber que algumas questões levantadas traziam preocupações comuns a todos os participantes e seguiam até mesmo uma determinada ordem cronológica. Esses resultados aparecem no quadro que se segue.

A importância desse grupo se fez notar, principalmente, pela oportunidade dos acompanhantes falarem de suas tensões e dificuldades, de compartilharem suas emoções com pessoas que vivenciaram a mesma situação vital e de se sentirem compreendidos e amparados por alguém da equipe.

\begin{tabular}{|c|c|c|}
\hline \multirow[b]{2}{*}{$1^{0}$} & Estágio do TMO & \multirow{2}{*}{$\begin{array}{l}\text { Repercussões psicológicas } \\
\text { Ansiedade frente ao que está por vir }\end{array}$} \\
\hline & Internação-isolamento & \\
\hline $2^{\circ}$ & Implantação do cateter & Ansiedade frente à cirurgia vascular \\
\hline $3^{0}$ & Início da quimioterapia & $\begin{array}{l}\text { Somatório dos sintomas somáticos e do medo das conseqüências da quimio- } \\
\text { terapia: alopecia, mucosite, aplasia. }\end{array}$ \\
\hline \multirow[t]{2}{*}{$4^{\circ}$} & Aplasia & Insegurança \\
\hline & & Sentimentos de persecutoriedade \\
\hline $5^{\circ}$ & Infusão da medula & Medo de "receber" as características do doador \\
\hline $6^{\circ}$ & Alopecia & Ansiedade frente a alteração da imagem corporal \\
\hline \multirow[t]{4}{*}{$7^{0}$} & Mucosite & Irritabilidade causada pela dor \\
\hline & & Impossibilidade de conversar ou se alimentar \\
\hline & & Dependência \\
\hline & & Impressão de que o tempo não passa \\
\hline $8^{\circ}$ & Pega da medula & Alívio, considerado o sucesso do tratamento \\
\hline \multirow[t]{3}{*}{$9^{\circ}$} & Alta & Insegurança \\
\hline & & Medo em não saber se cuidar sozinho \\
\hline & & Medo da necessidade de reinternação \\
\hline
\end{tabular}




\begin{tabular}{|c|c|c|}
\hline \multirow{3}{*}{19} & Percurso do grupo & Questões \\
\hline & Adaptação & O que faz um acompanhante? \\
\hline & & Ansiedade em não conseguir desempenhar o papel esperado \\
\hline \multirow[t]{3}{*}{2} & Repercussões do Diagnóstico & Impacto inicial \\
\hline & & Aceitação da doença \\
\hline & & Mudanças de vida: negativas e positivas \\
\hline \multirow[t]{4}{*}{30} & Repercussões do TMO & Decisão: Prós e contras \\
\hline & & Complicações e efeitos adversos \\
\hline & & Ansiedade pela pega \\
\hline & & Ansiedade pela alta \\
\hline \multirow[t]{3}{*}{$4^{\circ}$} & Medo da Recaída & Insegurança \\
\hline & & Medo de terem se sacrificado em vão \\
\hline & & Indecisão quanto a passar de novo ou não pela experiência \\
\hline \multirow[t]{4}{*}{$5^{\circ}$} & Questão da Morte & Crença no poder de cura da equipe \\
\hline & & Aparecimento da religiosidade \\
\hline & & Espera pelo milagre \\
\hline & & Resignação: Deus sabe o que faz \\
\hline
\end{tabular}

\section{4- FASE POSTERIOR AO TMO}

A qualidade de vida dos transplantados sofrerá as implicações do procedimento, obrigando-os a enfrentar as limitações físicas, o desenvolvimento de Doença do Enxerto Contra o Hospedeiro (DECH), a dor, a sensação de distorção da imagem corporal e as consequiências dos efeitos colaterais dos tratamentos: queda de cabelo, escurecimento da pele, emagrecimento ou edema ${ }^{(5)}$, além das alterações nos seus hábitos de vida, tais como a perda ou prejuízo da capacidade produtiva (trabalho e escola), das funções sexuais e da fertilidade (como efeito colateral de algumas quimioterapias ou radioterapias), bem como a perda da independência e de alguns papéis sociais ${ }^{(8)}$.

Em função dessas dificuldades de adaptação fora do contexto hospitalar, impôs-se a necessidade de uma intervenção psicológica após a alta da enfermaria. Essa intervenção ocorreu através de atendimentos individuais ambulatoriais
Trata-se da continuidade do atendimento da enfermaria até completados os cem dias pós-TMO. Esses atendimentos são realizados ambulatorialmente e sua freqüência varia em função da necessidade de cada paciente. Encerrado o período dos cem dias, optase pela alta, pelo retorno livre (no qual o paciente pode recorrer ao atendimento, quando sentir necessidade) ou por um encaminhamento para outro serviço que preste assistência psicológica.

\section{5- DISCUSSÃO}

A última década foi testemunha do crescimento de um novo campo de trabalho e pesquisa: a psiconcologia. Esse campo surge da necessidade de se oferecer apoio emocional ao paciente com câncer, assim como aos seus familiares e aos profissionais envolvidos no tratamento.

Apesar de se encontrar só recentemente implantada nos serviços hospitalares, a união entre a 
psicologia e a oncologia já tem produzido resultados amplamente reconhecidos através dos aumentos significativos da sobrevida, melhora da qualidade de vida e do fortalecimento psicológico para encarar a terminalidade $^{(2)}$.

A intervenção psicológica em uma Unidade de Transplante de Medula Óssea, se adequadamente estruturada, apresenta-se como um recurso que amplia os limites de ação da equipe médica no atendimento das necessidades que surgem em cada momento da trajetória do paciente oncológico: iniciando-se no diagnóstico, percorrendo o tratamento e podendo alcançar as situações posteriores de adaptação do paciente às seqüelas concretas ou subjetivas com que se deparam ${ }^{(3)}$.

Levando-se em consideração tais afirmações e conhecimentos organizados, buscou-se sistematizar um serviço de psicologia, na Unidade de Transplante de Medula Óssea do Hospital das Clínicas da Faculdade de Medicina de Ribeirão Preto, que se mostrasse o mais adequado do ponto de vista técnico, respeitan- do-se os limites e possibilidades concretas do contexto institucional em que ele se acha instalado.

Desse modo, procurou-se valorizar a necessidade de um acompanhamento psicológico que não se restringisse somente ao período de internação e ao paciente. Para tanto, o trabalho do profissional de saúde mental tem início no momento que precede a internação e se prolonga após a alta da enfermaria, além de se estender, também, aos familiares, especialmente ao acompanhante e ao doador.

Cabe aqui a ressalva de que se trata de um serviço aberto para reestruturações, tanto através da incorporação de novas modalidades quanto pela constante reformulação de outras, as que não se mostrarem eficientes para o atendimento dos objetivos do serviço. Daí a importância dos estudos realizados pela equipe de profissionais da área, como os referidos anteriormente, visando avaliar a pertinência e a resolutividade das intervenções oferecidas no âmbito da saúde mental.

TORRANO-MASETTI LM; OLIVEIRA ÉA \& SANTOS MA. Psychological service in a bone marrow transplantation unit. Medicina, Ribeirão Preto, 33: 161-169, apr./june 2000.

ABSTRACT: Bone marrow transplantation (BMT) represents an therapeutic alternative applied for the treatment of hematological malignancies as well as certain solid tumors and immunology disorders, when the conventional forms of treatment are not good choice. It is a complex procedure, both in the physical and psychological sense, and the need of a psychological intervention for the patient and families has been recognized by several authors in the literature. According to the literature, the relevance of the psychological counseling exceeds the period of hospitalization, including the pre-BMT and pos-BMT. More attention have to be dedicated to the families in this process. Considering these principles, a psychological support service was carried out in a large teaching hospital in Ribeirão Preto, Brazil (Hospital das Clínicas da Faculdade de Medicina de Ribeirão Preto) in order to provide support both for the patients and their families. The present study aims to describe the structure of this service, as well as to present some main datas obtained from some studies that investigated issues of avaliation, designed to assess the effectivity of some implemented psychological interventions before, during and after BMT. The results confirm available data in literature that underline the relevance of the pre-TMO intervention to the patient who had undergone a bone marrow transplantation, counseling and psychological support during the differents phases of the hospitalization and psychological support to the families.

UNITERMS: Psychology, Applied. Social Support. Bone Marrow Transplantation.

\section{REFERÊNCIAS BIBLIOGRÁFICAS}

1 - ROMANO WB. Princípios para a prática da psicologia clínica em hospitais. Casa do Psicólogo, São Paulo, 1999.

2 - CARVALHO MMJ. Psico-oncologia no Brasil: Resgatando o viver. Summus, São Paulo, 1998.
3 - VEIT MT; CHURARTZMANN \& BARROS MCM. O serviço de psico-oncologia do Hospital Israelita Albert Eisten. In: CARVALHO MMJ. Psico-oncologia no Brasil: resgatando o viver. Summus, São Paulo, p. 62-72, 1998.

4 - RIVERA LM. Blood cell transplantation: its impact on one family. Semin Oncol Nurs 13: 194-199, 1997. 
5 - LESKO LM. Bone Marrow Transplantation. In: HOLLAND J \& ROWLAND J, eds Handbook of psychooncology: psychological care for the patient with cancer. Oxford University Press, New York, p.163-172, 1990.

6 - HIGGINSON I. Advanced cancer: aiming for the best in care. Qual Health Care 2: 112-116, 1993

7 - HOLLAND J \& ROWLAND JH, eds Handbook of psychooncology. Oxford University Press, New York, 1990.

8 - COOPER MC \& POWELL G. Technology and care in a bone marrow transplantation unit: creating and assuaging vulnerability. Holist Nurs Pract 12: 57-62, 1998.

9 - KRISTJANSON LJ; NIKOLETTI S; POROCK D; SMITH M; LOBCHUK M \& PEDLER P. Congruence between patients and family caregiver's perceptions of symptom distress in patients whith terminal cancer. J Palliat Care 14: p. 24-32, 1998.

10 - BUCKNER CD; PETERSEN FB \& BOLONESI BA. Bone marrow donors. In: FORMAS SJ; BLUME KG \& THOMAS ED, eds. Bone marrow transplantation, Blackwell Scientific Publication, Boston, p. 259-265, 1994.
11 - TORRANO-MASETTI LM; OLIVEIRAEA; SANTOS, MA; SIMÕES BP \& SPONHOLZ Jr. A. Saúde mental e transplante de medula óssea: um estudo retrospectivo. In: Resumos, XXIX Reunião Anual da Sociedade Brasileira de Psicologia (SBP), Campinas-SP, p. 248, 1999.

12 - WELLISH DK \& WOLCOTT DL. Psychological issues in bone marrow transplantation. In: FORMAS SJ; BLUME KG. \& THOMAS ED, eds. Bone marrow transplantation, Blackwell Scientific Publication, Boston, p. 556-571, 1994.

13 - TORRANO-MASETTI LM; OLIVEIRA EA; SIMÕES BP \& SANTOS, MA. Grupo preparatório para pacientes na fila de espera para transplante de medula óssea: avaliação de uma estratégia de intervenção. In: Resumos, XXIX Reunião Anual da Sociedade Brasileira de Psicologia (SBP), Campinas-SP, p. 248, 1999.

14 - OLIVEIRA EA; TORRANO-MASETTI LM; SANTOS MA \& SIMÕES BP. Acompanhando o acompanhante. In: Resumos, XXIX Reunião Anual da Sociedade Brasileira de Psicologia (SBP), Campinas-SP, p. 268, 1999.

Recebido para publicação em 27/01/2000

Aprovado para publicação em 20/06/2000 